\title{
Andere spezifische Diabetesformen und exokrine Pankreasinsuffizienz (Update 2019)
}

\author{
Susanne Kaser · Yvonne Winhofer-Stöckl · Lili Kazemi-Shirazi · Sabine E. Hofer · Helmut Brath · Harald Sourij · \\ Greisa Vila · Heidemarie Abrahamian - Michaela Riedl · Raimund Weitgasser · Michael Resl · Martin Clodi · \\ Anton Luger
}

Online publiziert: 12. April 2019

(C) Der/die Autor(en) 2019

\begin{abstract}
Zusammenfassung Die unter der Kategorie ,andere spezifische Diabetesformen" zusammengefassten Störungen des Glukosestoffwechsels stellen pathophysiologisch und therapeutisch eine sehr heterogene Krankheitsgruppe dar. Umfasst werden Diabetesformen, die im Rahmen von anderen endokrinologischen Erkrankungen auftreten (z.B. Akromegalie, Cushing-Syndrom), pankreoprive Formen (z. B. postoperativ, Pankreatitis, Pankreastumoren, Hämochromatose, zystische Fibrose), medikamentös induzierte Diabetesformen (z. B. Antipsychotikatherapie, Glukokortikoidtherapie, HAART), genetische Formen (z.B. i.R. eines MODY, neonataler Diabetes, Down-Syndrom, Klinefelter-Syndrom, Turner-Syndrom), Infektionen (z. B. kongenitale Rötelninfektion) und seltene autoimmune Formen (z. B. Stiffman-Syndrom). Nicht nur pankreoprive Formen, sondern auch Typ 1 oder langjähriger Typ 2 Diabetes mellitus sind häufig mit einer exokrinen Pankreasinsuffizienz assoziiert.
\end{abstract}

\footnotetext{
S. Kaser $(\bowtie)$

Department für Innere Medizin I, Medizinische Universität Innsbruck, Anichstraße 35, 6020 Innsbruck, Österreich susanne.kaser@i-med.ac.at

Christian Doppler Labor für Insulinresistenz, Medizinische Universität Innsbruck, Innsbruck, Österreich

Y. Winhofer-Stöckl · G. Vila · M. Riedl · A. Luger Klinische Abteilung für Endokrinologie und Stoffwechsel, Universitätsklinik für Innere Medizin III, Medizinische Universität Wien, Wien, Österreich

L. Kazemi-Shirazi

Klinische Abteilung für Gastroenterologie und Hepatologie, Universitätsklinik für Innere Medizin III, Medizinische Universität Wien, Wien, Österreich

\section{S. E. Hofer}

Department für Pädiatrie I, Medizinische Universität

Innsbruck, Innsbruck, Österreich
}

Schlüsselwörter Andere spezifische Diabetesformen . Exokrine Pankreasinsuffizienz - Pankreoprive Diabetesformen

\section{Other specific types of diabetes and exocrine pancreatic insufficiency (Update 2019)}

Summary The heterogenous catagory "specific types of diabetes due to other causes" encompasses disturbances in glucose metabolism due to other endocrine disorders such as acromegaly or hypercortisolism, drug-induced diabetes (e.g. antipsychotic medications, glucocorticoids, immunosuppressive agents, highly active antiretroviral therapy (HAART)), genetic forms of diabetes (e.g. Maturity Onset Diabetes of the Young (MODY), neonatal diabetes, Down Syndrome, Klinefelter Syndrome, Turner Syndrome), pancreatogenic diabetes (e.g. postoperatively, pancreatitis, pancreatic cancer, haemochromatosis, cystic fibro-

H. Brath

Gesundheitszentrum Wien-Süd, Wien, Österreich

H. Sourij

Klinische Abteilung für Endokrinologie und Diabetologie,

Universitätsklinik für Innere Medizin, Medizinische

Universität Graz, Graz, Österreich

H. Abrahamian

Internistisches Zentrum Baumgartner Höhe,

Otto-Wagner-Spital, Wien, Österreich

R. Weitgasser

Abteilung für Innere Medizin, Privatklinik

Wehrle-Diakonissen, Salzburg, Österreich

M. Resl · M. Clodi

Abteilung für Innere Medizin, Konventhospital der

Barmherzigen Brüder Linz, Linz, Österreich 
sis), and some rare autoimmune or infectious forms of diabetes. Diagnosis of specific diabetes types might influence therapeutic considerations. Exocrine pancreatic insufficiency is not only found in patients with pancreatogenic diabetes but is also frequently seen in type 1 and long-standing type 2 diabetes.

Keywords Other specific types of diabetes - Exocrine pancreatic insufficiency $\cdot$ Pancreatogenic diabetes

\section{Diabetesformen im Rahmen anderer endokriner Erkrankungen}

Endokrinologische Erkrankungen, die mit Glukosestoffwechselstörungen einhergehen, und deren diagnostische Tests sind in Tab. 1 dargestellt.

\section{Cushing-Syndrom}

In der überwiegenden Mehrzahl ist ein Cushing-Syndrom auf eine vermehrte ACTH-Produktion zurückzuführen. Diese wiederum tritt am häufigsten bei ACTHsezernierenden Hypophysenadenomen auf, seltener sind ektope ACTH-Quellen bei meist malignen Tumoren (z.B. Bronchialkarzinom, Thymuskarzinoid). Die häufigste Ursache für ACTH-unabhängige Cushing Syndrome sind Nebennierenadenome, seltener Nebennierenkarzinome oder mikro- oder makronoduläre adrenale Hyperplasien [1]. Die klinischen Symptome ähneln teils den diagnostischen Kriterien eines metabolischen Syndroms. Neben einer Gewichtszunahme, einer gestörten Glukosetoleranz bis zum Auftreten eines Diabetes mellitus und einer arteriellen Hypertonie finden sich häufig osteoporotische Wirbelkörperfrakturen, ein Hypogonadismus und Hautveränderungen, insbesondere rote Striae (Striae rubrae), eine Myopathie und auch psychiatrische Auffälligkeiten [1]. Die Therapie erfolgt sofern möglich chirurgisch, je nach Ätiologie finden ansonsten Dopamin-Agonisten oder das Somatostatinanalogon Pasireotid (Hypophysenadenome) oder adrenostatisch bzw. adrenolytisch wirkende Medikamente (ektope oder adrenale Formen) bzw. strahlentherapeutische Verfahren Anwendung (hypophysäre Formen) [2]. Bezüglich therapeutischer Überlegungen s. medikamentös-induzierte Diabetesformen.

\section{Akromegalie}

Zum ganz überwiegenden Teil ist eine überschießende Sekretion von Wachstumshormon bei Hypophysenadenomen Ursache einer Akromegalie, nur sehr selten ist eine ektope GHRH-Sekretion für eine Akromegalie verantwortlich. Neben unspezifischen Symptomen wie Cephalea, Hyperhidrosis, Schlafapnoe, Hypertonie und Arthralgien sind akrales Wachstum und Makroglossie charakteristische Symptome einer Akromegalie. Begleitet werden diese von metabolischen Komplikationen in Form einer Glukosetoleranzstörung bzw. eines manifesten Diabetes mellitus sowie einer Dyslipidämie [3]. Die Serum IGF-1 Konzentration wird zum Screening herangezogen, wobei ein präexistenter Diabetes mellitus ebenso wie katabole Zustandsbilder oder eine eingeschränkte Leberfunktion zu falsch negativen Ergebnissen führen können. Bestätigt wird das Vorliegen einer Akromegalie mittels eines $75 \mathrm{~g}$ oralen Glukosetoleranztests (OGTT) (GH Nadir <1,0 ug/l bzw. <0,4 ug/l). Bei unklaren diagnostischen Konstellationen kann bei Patienten mit diabetischer Stoffwechsellage ein dynamischer Test mit Galanin oder Thyreotropin-Releasing-Hormone erwogen werden [4].

Therapeutisch ist eine chirurgische Sanierung anzustreben, sollte diese nicht möglich bzw. nicht erfolgreich sein, kommen bei milden Formen Dopaminagonisten, üblicherweise jedoch Somatostatinanaloga oder der GH-Rezeptor-Antagonist Pegvisomant zum Einsatz, ggf. auch strahlentherapeutische Verfahren.

Pathophysiologisch führt eine kontinuierliche exzessive Erhöhung der GH-Konzentration $\mathrm{zu}$ einer Störung im Insulinsignaltransduktionsweg klinisch einer Insulinresistenz entsprechend. Die Therapie eines Akromegalie-assoziierten Diabetes unterscheidet sich prinzipiell nicht von der eines Diabetes mellitus Typ 2.

\section{Andere mit DM-assoziierte Endokrinopathien}

Ein primärer Hyperaldosteronismus, ebenso wie ein Phäochromozytom, eine manifeste Hyperthyreose oder die seltenen neuroendokrinen Neoplasien Glukagonom und Somatostatinom können mit Störungen des Glukosestoffwechsels einhergehen (Tab. 1).
Tab. 1 Endokrinologische Erkrankungen mit erhöhtem Risiko für das Auftreten einer Glukosetoleranzstörung sowie empfohlene Screeninguntersuchungen bzw. diagnostische Tests. (Adaptiert nach [35])

\begin{tabular}{|l|l|}
\hline $\begin{array}{l}\text { Erkrankung } \\
\text { Cushing Syndrom }\end{array}$ & $\begin{array}{l}\text { Screening und Diagnostik } \\
\text { 1 } \mathrm{mg} \text { Dexamethason Suppressionstest }\end{array}$ \\
\hline Akromegalie & Serum IGF-1, 75g OGTT: GH Nadir \\
\hline Hyperthyreose & TSH, fT4, fT3 \\
\hline Phäochromozytom & Serum oder Harn Metanephrin/Normetanephrin \\
\hline Primärer Hyperaldosteronismus & Renin/Aldosteron Ratio \\
\hline Glukagonom & Plasma Glukagon \\
\hline Somatostatinom & Plasma Somatostatin \\
\hline IGF-1 Insulin like growth factor-1, OGTT oraler Glukosetoleranztest, TSH Thyreotropin \\
\hline
\end{tabular}


Tab. 2 Medikamente, für die in unterschiedlicher Ausprägung eine Beeinträchtigung des Glukosestoffwechsels beschrieben wurde [13-15, 35, 96-102]

\begin{tabular}{l|l|}
\hline Antiinfektiva & Fluoroquinolone (Moxifloxacin) \\
\hline & $\begin{array}{l}\text { Antiretrovirale HIV-Therapie (Proteaseinhibitoren, NRTIs) } \\
\text { Pentamidin }\end{array}$ \\
\hline Antipsychotika & $\begin{array}{l}\text { Erstgenerationsantipsychotika (Chlorpromazin, Perphenazin, andere Phenothiazine) } \\
\text { Zweitgenerationsantipsychotika (Clozapin, Iloperidon, Olanzapin, Paliperidon, Quetiapin, Risperi- } \\
\text { don) }\end{array}$ \\
\hline Anithypertensiva & Betablocker (Atenolol, Metoprolol, Propranolol) \\
\hline Vasopressoren & Thiaziddiuretika (Hydrochlorothiazid, Chlorthalidon, Chlorothiazid, Indapamid) \\
\hline Hormone & Ginephrin, Norepinephrin \\
\hline & Orale Kontrazeptiva (Östrogen-Progestagen Kombinationspräparate, Progestin Monopräparate) \\
\hline Progestin (Megestrolacetat) \\
\hline Wachstumshormon, Tesamorelin \\
\hline Lipidsenker & Thyroxin \\
\hline Immunsuppressiva & Nikotinsäurederivate, Statine \\
\hline Antikörper & Tacrolimus, Cyclosporin A, Sirolimus \\
\hline Andere & Pembrolizumab, Nivolumab, Ipilimumab \\
\hline
\end{tabular}

\section{Medikamentös-induzierte Diabetesformen}

Zahlreiche Medikamente führen in unterschiedlicher Ausprägung zu einer Beeinträchtigung des Glukosestoffwechsels, wichtige Vertreter sind in Tab. 2 zusammengefasst.

Neben Glukokortikoiden sind insbesondere verschiedene typische, aber auch atypische Antipsychotika - unter anderem bedingt durch eine gesteigerte Nahrungszufuhr und entsprechender Gewichtszunahme - mit einem erhöhten Diabetesrisiko verbunden (siehe Kapitel Abrahamian et al., Psychische Erkrankungen und Diabetes mellitus) [5].

Jegliche systemische Glukokortikoidtherapie ist mit einer erhöhten Diabetesinzidenz verbunden. Das Risiko für das Auftreten einer Hyperglykämie ist vor allem bei $>65$-jährigen Patienten, zugrundeliegender Glukosestoffwechselstörung, reduzierter Nierenfunktion und rheumatologischen bzw. nephrologischen Patienten in Abhängigkeit von der Glukokortikoiddosis und Therapiedauer signifikant erhöht [6, 7]. Charakterisiert ist ein Glukokortikoid-induzierter Diabetes bei morgendlicher Glukokortikoidgabe durch eine meist normale Nüchternglukosekonzentration und eine ausgeprägte Hyperglykämie untertags. Infolgedessen sind postprandiale Glukosemessungen zum Screening und zur Monitorisierung besser geeignet als alleinige Nüchternglukosemessungen [8]. Der HbAlc Wert eignet sich nur bei längerfristiger chronischer Glukokortikoidtherapie zur Therapieüberwachung.

Die diabetogene Stoffwechsellage ist bei Hyperkortisolismus neben einer meist ausgeprägten Gewichtszunahme auch auf eine hepatische und periphere Insulinresistenz sowie eine verminderte Insulinsekretion zurückzuführen [9]. Die Behandlung unterscheidet sich nicht von der eines Diabetes mellitus Typ 2. Sie richtet sich nach Pharmakokinetik des ver- wendeten Glukokortikoidpräparates und dem daraus resultierenden Blutzuckertagesprofil sowie den Komorbiditäten des Patienten. Als Ziel, insbesondere im stationären Bereich, gilt es, einen Blutzucker von $<180 \mathrm{mg} / \mathrm{dl}$ zu erreichen.

Eine besondere Diabetesform stellt der sogenannte Posttransplantationsdiabetes (NODAT: new onset of diabetes after transplantation) dar. Die Prävalenz wird abhängig von dem transplantierten Organ langfristig mit bis zu $40 \%$ angegeben [10, 11]. Neben Glukokortikoiden weisen vor allem Calcineurin Inhibitoren (insbesondere Tacrolimus) ein diabetogenes Potential auf. Die Behandlung des NODAT unterscheidet sich zwar grundsätzlich nicht von der eines Diabetes mellitus Typ 2, die Therapieoptionen sind jedoch häufig aufgrund der Komorbiditäten eingeschränkt.

HAART, insbesondere Proteaseinhibitoren und Nukleosidische Reverse-Transkriptase-Inhibitoren (NRTIs), kann gemeinsam mit der Inflammation einer chronischen HIV-Infektion diabetogen wirken. So zeigte eine rezente Arbeit an 522 österreichischen Patienten mit chronischer HIV-Infektion, dass $50 \%$ der im Schnitt 42-jährigen Teilnehmer insulinresistent waren [12]. Die Wahl metabolisch neutraler antiretroviraler Medikation, regelmäßige Kontrollen metabolischer Parameter und ggf. frühzeitige Therapie scheinen ratsam.

Antikörper wie Pembrolizumab, Nivolumab und Ipilimumab, eingesetzt in der Tumortherapie, können zu einer $\beta$-Zell-Zerstörung in Form eines Autoimmundiabetes führen [13-15].

In der Gruppe der Antihypertensiva weisen vor allem Thiaziddiuretika und nicht-vasodilatierend wirkende Betablocker ein mildes diabetogenes Potential auf [16]. Eine Statintherapie ist vor allem bei Hochrisikopatienten mit einer erhöhten Diabetesinzidenz ver- 
Tab. 3 Übersicht über genetische Diabetesformen. (Adaptiert von [35])

\begin{tabular}{l|l|}
\hline $\begin{array}{l}\text { Genetische Störungen der } \\
\text { Betazellfunktion }\end{array}$ & MODY \\
& $\begin{array}{l}\text { Transienter oder permanenter } \\
\text { neonataler Diabetes }\end{array}$ \\
\hline & Mitochondriale Diabetesformen \\
\hline Andere \\
\hline $\begin{array}{l}\text { Genetische Störungen der } \\
\text { Insulinwirkung }\end{array}$ & Typ A Insulinresistenz \\
\hline & Leprechaunismus \\
\hline & Rabson-Mendenhall-Syndrom \\
\hline & Lipoatropher Diabetes \\
\hline Mit Diabetes mellitus assoziierte & Andere \\
\hline andere genetische Erkrankungen & Down-Syndrom \\
\hline & Klinefelter-Syndrom \\
\hline & Turner-Syndrom \\
\hline Wolfram-Syndrom \\
\hline Friedreich-Ataxie \\
\hline Chorea Huntington \\
\hline Laurence-Moon-Biedl-Syndrom \\
\hline Dystrophia myotonica \\
\hline Porphyrie \\
\hline Prader-Willi-Syndrom \\
\hline Andere \\
\hline
\end{tabular}

bunden, wobei der kardiovaskuläre Benefit dem metabolischen Risiko um ein Vielfaches überlegen ist [17].

\section{Genetische Diabetesformen}

Unter genetischen Formen wird ein heterogenes Krankheitsbild zusammengefasst. Am häufigsten handelt es sich um erblich bedingte Diabetesformen, zurückzuführen auf eine $\beta$-Zell-Dysfunktion (MODY) oder aber eine Glukosestoffwechselstörung im Rahmen von anderen genetischen Erkrankungen. Selten sind genetische Störungen der Insulinwirkung (Tab. 3).

\section{MODY}

Beim Maturity Onset Diabetes of the Young (MODY) handelt es sich um eine monogenetische Diabetesform mit unterschiedlicher Ausprägung einer Betazelldysfunktion, aber erhaltener Insulinsensitivität, die bis $\mathrm{zu} 2 \%$ aller Menschen mit Diabetes betrifft. Der genetische Defekt ist heterogen und beeinflusst die Therapienotwendigkeit und -form. MODY Formen werden autosomal dominant vererbt, am häufigsten sind in unseren Breiten Mutationen der Transkriptionsfaktoren HNF1 $\alpha$ und HNF4 $\alpha$ und des Glucokinasegens (GCK), die zusammen mehr als $80 \%$ aller MODY Fälle ausmachen. Ein Screening sollte bei Patienten unter 25 Jahren mit positiver Familienanamnese und negativem Autoantikörperstatus erfolgen [18-21]. Aufgrund der therapeutischen und prognostischen Relevanz sollte die Diagnose mittels genetischer Ana- lyse gesichert werden. Die Wahrscheinlichkeit für das Vorliegen einer monogenetischen Diabetesform kann online ermittelt werden: https://www.diabetesgenes. org/mody-probability-calculator/.

\section{Glucokinase (MODY 2)}

Die bei uns zweithäufigste MODY-Form erklärt sich durch eine erhöhte Glukoseschwelle für die Insulinsekretion infolge einer Mutation im GlucokinaseGen. Folge ist eine über viele Jahre asymptomatische, meist mild bis moderate Erhöhung des Nüchternblutzuckers. Charakteristisch ist neben der erhöhten Fastenglukose ein nur milder Blutzuckeranstieg im oralen Glukosetoleranztest [22]. Entsprechend ist der HbAlc Wert meist über Jahre stabil und nur leicht bis max. 8\% (64 mmol/mol) erhöht [23]. Die Patienten sind überwiegend asymptomatisch und fallen durchwegs im Rahmen von Screening Untersuchungen auf [24]. Eine medikamentöse Therapie ist - sofern keine Schwangerschaft vorliegt - meist nicht notwendig [25], da das Risiko für mikro- und makrovaskuläre Folgeerkrankungen in Beobachtungsstudien nicht erhöht scheint [23, 26]. Einen besonderen Stellenwert hat die genetische Diagnosesicherung einer Glucokinase-Mutation in der Schwangerschaft: Weist der Fetus einer Glukokinase-Mutationsträgerin selbst keine Mutation auf, besteht aufgrund der als Folge der maternalen Hyperglykämie vermehrten Insulinsekretion ein erhöhtes Risiko für eine Makrosomie. Im Gegensatz dazu weisen Kinder von Vätern mit einer Glukokinase-Mutation bei negativem Carrierstatus der Mutter ein vermindertes Geburtsgewicht auf [27, 28].

\section{HNF1 $\alpha$ (MODY 3)}

Die häufigste MODY Form manifestiert sich in Abhängigkeit von der Lokalisation der Mutation zum Großteil vor dem 25. Lebensjahr [24]. Zugrunde liegt eine Betazell-Dysfunktion, die konsekutiv zu Betazellversagen und einer Hyperglykämie führt. Laborchemische Besonderheiten bei dieser Diabetesform sind eine verminderte Glukose-Nierenschwelle, einhergehend mit Glukosurie auch bei noch nicht erkrankten Mutationsträgern und ein sehr niedriges high sensitive (hs) CRP sowie deutlich erhöhte postprandiale Werte im oralen Glukosetoleranztest [21, 29]. Charakteristisch ist ein sehr sensitives Ansprechen dieser Patienten auf eine Sulfonylharnstofftherapie [30].

\section{HNF4 $\alpha$ (MODY 1)}

Die pathophysiologische und klinische Präsentation dieser selteneren MODY Form ( $4 \%$ aller monogenetischen Diabetesformen) ist einem HNF1 $\alpha$ Mutationen bedingten Diabetes ähnlich. Die Manifestation erfolgt durch $\beta$-Zell-Defizienz meist vor dem 25. Lebensjahr, auch diese Betroffenen sprechen meist sehr gut auf eine Sulfonylharnstofftherapie an [24, 31]. Charakteristischerweise ist das Geburtsgewicht von Mutationsträgern hoch [32]. 


\section{HNF1ß (MODY 5)}

Neben einer progredienten Betazelldysfunktion ist eine HNF1 $\beta$-Mutation durch Malformationen im Urogenitaltrakt (z.B. Nierendysplasie, Nierenzysten, hypoplastische glomerulozystische Nierenerkrankung) und ein atrophes Pankreas gekennzeichnet, zudem besteht häufig ein niedriges Geburtsgewicht [33]. Spontanmutationen sind bei dieser monogenetischen Diabetesform häufig [24]. Meist ist eine Insulintherapie erforderlich [34].

\section{Andere seltene MODY Formen}

Die seltene IPF-1 Mutation (MODY 4) geht mit Pankreasagenesie und neonatalem Diabetes einher und erfordert entsprechend eine Insulintherapie [34]. Weitere MODY Mutationen wurden unter anderem im Carboxylester-Lipase-Gen (MODY 7) oder NeuroD1-Gen (MODY 6) beschrieben [35, 36].

\section{Mitochondriale Diabetesformen}

Mitochondriale Diabetesformen sind - sofern es sich um keine Spontanmutation handelt - maternal vererbt und häufig mit neuromuskulären Symptomen sowie Hörstörungen verbunden, wobei letztere häufig der Diabetesmanifestation vorausgehen. Laut DPVRegister machen diese Formen weniger als $1 \%$ aller Diabetesfälle im Erwachsenenalter aus [37]. Den beiden Syndromen „maternally inherited diabetes and deafness“ (MIDD) und "mitochondrial encephalomyopathy with lactic acidosis and stroke-like episodes" (MELAS) liegt die gleiche Genmutation zugrunde [38], vermutlich aufgrund der verschieden ausgeprägten Heteroplasmie kommt es jedoch zu unterschiedlicher Symptomatik [39]. Das DIDMOAD- oder WolframSyndrom ist charakterisiert durch das Auftreten eines Diabetes insipidus, eines Diabetes mellitus, einer Optikusatrophie und Taubheit. Eine spezifische Therapie der mitochondrialen Diabetesformen existiert nicht, beim MELAS Syndrom werden L-Arginin und auch Carnitin bzw. Coenzym Q10 zur Therapie der neurologischen Symptomatik versuchsweise eingesetzt [40].

\section{Neonataler Diabetes}

Neonataler Diabetes ist definiert als Diabetesmanifestation vor dem 6. Lebensmonat, wobei transiente von permanenten Verläufen unterschieden werden. Ein Diabetes-Relaps bei transienten Verlaufsformen wird in bis zu $50 \%$ der Fälle beschrieben [41]. In Österreich beläuft sich die Inzidenz auf 1/230.000 für transiente und $1 / 530.000$ für permanente neonatale Diabetesformen [42]. Die Ursache ist meist monogenetisch, wobei 6q24 Abnormitäten (uniparentale Disomie, Duplikation, Methylierungsdefekte), Mutationen an Untereinheiten des ATP abhängigen Kalium-Kanals (ABCC8, KCNJ11) sowie Mutationen am Proinsulin codierenden INS-Gen, Glukokinase-Gen
GCK, HNF1 $\beta$ ursächlich beschrieben wurden [43]. Neonataler Diabetes kann auch im Rahmen syndromaler Erkrankungen auftreten, wobei IPEX-Syndrom (FOXP3), Wolcott-Rallison-Syndrom (EIF2AK3), Pankreasagenesie (PDX1, PTF1A) ca. $10 \%$ der neonatalen Diabetesfälle erklären. Die genetische Abklärung ist aufgrund therapeutischer Konsequenzen wichtig. Die initiale Behandlung mit Insulin unter Vermeidung von Hypoglykämien ist immer indiziert, wobei die Verwendung einer Insulinpumpe zur Applikation der geringen Insulinmengen bevorzugt Einsatz finden sollte. Eine sensorunterstütze Pumpentherapie ist bei ausreichend subkutanem Fettgewebe ab einem Körpergewicht von ca. $3 \mathrm{~kg}$ möglich. Eine orale Behandlung mit Sulfonylharnstoffen ist indiziert, wenn Mutationen an den Untereinheiten SUR1 und Kir6.2 des ATP abhängigen Kaliumkanals als Ursache für den vorliegenden neonatalen Diabetes genetisch bestätigt wurden [44].

\section{Pankreopriver Diabetes}

Generell kann jede Erkrankung, die zu einer diffusen Zerstörung des Pankreasgewebes führt, mit einem pankreopriven Diabetes einhergehen. In westlichen Ländern sind pankreoprive Ursachen für etwa 5-10\% aller Diabeteserkrankungen verantwortlich [45]. Ätiologisch kommen dafür traumatische oder infektiöse Ursachen ebenso wie Pankreasoperationen, Pankreasagenesie, Pankreaskarzinome oder Pankreatitiden in Frage. Letztere sind für etwa $3 / 4$ aller pankreopriven Diabetesformen verantwortlich [46]. Pankreaskarzinome stellen insofern eine Ausnahme dar, als sie auch bei lokalisiertem Vorliegen ohne diffuse Zerstörung des Pankreasgewebes zu einer diabetogenen Stoffwechsellage führen können, der Grund dafür ist bisher nicht klar [47]. Hereditäre Stoffwechselerkrankungen wie Zystische Fibrose und Hämochromatose können ebenso zu pankreoprivem Diabetes führen. Auch die seltene und primär in asiatischen Ländern vorkommende fibrokalkulöse Pankreatopathie (FCPP) kann sekundär einen Diabetes mellitus bedingen $[35,36]$.

Bei postoperativen Patienten ist das Risiko für die Entwicklung eines pankreopriven Diabetes abhängig von der Art der Operation. Naturgemäß beträgt das Risiko $100 \%$ bei total pankreatektomierten Patienten, bei Patienten mit Whipple Operation (partielle Pankreatikoduodenektomie) wird das Risiko in älteren Studien mit $26 \%$ beziffert, wobei ein beträchtlicher Teil der damals untersuchten Patienten schon präoperativ an einem Diabetes erkrankt war [48].

Diagnostische Kriterien eines pankreopriven Diabetes umfassen eine Betazelldysfunktion, das Fehlen von Autoimmunantikörper und das Vorliegen einer Erkrankung des exokrinen Pankreas (Diagnostik s. unten) [49]. Die betroffenen Patienten weisen typischerweise eine hohe Insulinsensitivität auf, vorausgesetzt, dass dem pankreoprivem Diabetes kein Diabetes mel- 
litus Typ 2 oder Prädiabetes mit Insulinresistenz vorausgegangen ist.

Eine zufriedenstellende glykämische Kontrolle ohne Auftreten von klinisch signifikanten Hypoglykämien kann sich bei pankreoprivem Diabetes als schwierig herausstellen. Ursache dafür sind das Fehlen der gegenregulatorisch wirkenden Hormone Somatostatin und Glukagon ebenso wie eine durch die Grundkrankheit bedingte Malabsorption. Hinzu können schlechte Compliance und Lifestyle-Problematik bei Patienten mit alkoholischer Pankreatitis kommen [49].

Die systemische Insulinsensitivität ist bei pankreoprivem Diabetes typischerweise normal oder sogar erhöht, die hepatische Insulinsensitivität kann auch vermindert sein. Zur Therapie des pankreopriven Diabetes liegen keine spezifischen internationalen Richtlinien vor. Eine Metformintherapie kann bei gleichzeitig bestehender Insulinresistenz $\mathrm{zu}$ einer Verbesserung der Glykämie führen, allerdings wird Metformin aufgrund der gastrointestinalen Nebenwirkungen von diesem Patientenkollektiv meist schlecht toleriert. Bei hoher Insulinsensitivität ist der Effekt auf die Glykämie zudem als begrenzt anzunehmen, allerdings belegen Studien ein deutlich vermindertes Pankreaskarzinomrisiko unter Metformintherapie [50]. Inkretin-basierte Therapien sollten aufgrund eines potentiell erhöhten Pankreatitisrisikos bis zur endgültigen Klärung der Sicherheit der Wirkstoffe primär nicht zur Therapie eines pankreopriven Diabetes herangezogen werden. Sulfonylharnstoffe oder Glinide sind grundsätzlich geeignet, die Insulinsekretion bei vorhandener Betazellrestfunktion zu steigern, der Einsatz von Sulfonylharnstoffen ist allerdings aufgrund des besonderen Hypoglykämierisikos dieser Patienten streng zu monitieren. SGLT-2-Hemmer sind aufgrund des vorliegenden absoluten Insulinmangels und des damit verbunden erhöhten Risikos einer euglykämischen Ketoazidose nicht zu empfehlen. Therapie der Wahl ist daher bei einem Großteil der betroffenen Patienten Insulin, optimalerweise in Form eines Basis-Bolus-Regimes.

\section{Diabetes bei zystischer Fibrose (CFRD)}

Diabetes mellitus bei zystischer Fibrose (engl. cystic fibrosis related diabetes, CFRD) stellt die häufigste extra-pulmonale Komplikation bei Patienten mit zystischer Fibrose dar. Die Prävalenz steigt mit zunehmendem Patientenalter und liegt bei Erwachsenen $40-50 \%$ [51], bei Patienten nach Organtransplantation (Lungen- oder Lebertransplantation) vermutlich noch höher.

Pathophysiologisch steht vor allem die verminderte Insulinsekretion durch die krankheitsspezifische Pankreasfibrose im Vordergrund. Einige Autoren beschreiben zudem eine variable Insulinresistenz durch rezidivierende Infekte und wiederholte Glukokortikoidtherapie im Rahmen von Infektexazerbationen [52,
53]. Der CFRD ist assoziiert mit einer Verschlechterung der Lungenfunktion, des Ernährungsstatus sowie letztendlich des Überlebens [54, 55].

Als Screening-Methode wird die Durchführung eines oralen Glukosetoleranztests jährlich ab dem 10. Lebensjahr unter stabilen klinischen Bedingungen empfohlen [56]. Zusätzlich wird ein Screening in bestimmten klinischen Situationen empfohlen: während eines stationären Aufenthalts aufgrund einer Infektexazerbation (Erstellung eines Glukoseprofils mit Nüchtern- und postprandialen Werten), bei enteraler Ernährung sowie bei Frauen mit Kinderwunsch vor der geplanten Schwangerschaft, zwischen der 11. und 13. sowie zwischen der 24. und 28. Schwangerschaftswoche, und 6-12 Wochen nach der Entbindung.

Als Grenzwerte für die Diagnose eines CFRD gelten die allgemeinen Grenzwerte zur Diabetes-Diagnose. Zudem wurde wiederholt ein erhöhter 1-Stunden postprandialer Wert $>200 \mathrm{mg} / \mathrm{dl}$ mit einer klinischen Verschlechterung in Zusammenhang gebracht, weswegen bei Patienten mit dieser Form der Glukoseintoleranz (indeterminate glucose tolerance (INDET)) in Situationen klinischer Verschlechterung eine vorübergehende Insulintherapie in Erwägung gezogen werden sollte.

HbAlC und Nüchternglukose sind wiederholt als nicht geeignet für die Diagnose des CFRD beschrieben worden [53]. Der Einsatz der kontinuierlichen Glukosemessung (CGMS) wurde in einigen Studien empfohlen, ist aber als generelle Screening-Methode noch nicht etabliert. Sie sollte jedoch bei Patienten mit klinischer Verschlechterung und Hinweis auf CFRD, jedoch normalem OGTT, eingesetzt werden.

Die Therapie der Wahl ist jegliche Insulintherapie, wobei sämtliche Therapieformen in Frage kommen: Der Insulinbedarf ist meist niedriger als bei Patienten mit Typ 1 Diabetes und wird mit 0,38 IE $/ \mathrm{kg} / \mathrm{Tag}$ bei Jugendlichen, 0,46 IE/kg/Tag bei Erwachsenen und 0,58 IE/kg/Tag bei Patienten nach Organtransplantation angegeben [57]. Studien mit oralen Antidiabetika, insbesondere Sulfonylharnstoffen, konnten nur einen vorübergehenden Effekt zeigen und werden derzeit nicht empfohlen. Hinsichtlich diätischer Empfehlungen unterscheidet sich der CFRD wesentlich von anderen Diabetesformen, da sowohl von einer Kalorien-, Kohlenhydrat-, und Protein-reduzierten Diät abgeraten und eine Protein- und Salz-reiche Ernährung sogar empfohlen wird [56]. Lediglich „Softdrinks“ sollen in der Ernährung weggelassen werden, da sie zu teils schwer beherrschbaren Blutzuckeranstiegen führen [58].

Die Verlaufskontrolle unterscheidet sich wesentlich von anderen Diabetesformen. Als primärer Parameter gilt der Gewichtsverlauf. Jeder ungewollte Gewichtsverlust bei Patienten mit CFRD soll Anlass für eine Therapiekontrolle geben. Ergänzend kann das HbAlC herangezogen werden, wobei ein HbAlC im Normbereich, also $<6 \%$, zumindest $<6,5 \%$, angestrebt werden sollte. 
Neben den bereits beschriebenen Komplikationen des CFRD (Gewichtsverlust, Verschlechterung des Ernährungszustandes und der Lungenfunktion) spielen von den klassischen diabetischen Spätkomplikationen vor allem mikroangio- und neuropathische Komplikationen eine Rolle, die aufgrund der in den letzten Jahrzehnten deutlich gesteigerten Lebenserwartung bei CF zunehmen werden. Deshalb wird ein jährliches Screening beginnend 5 Jahre nach DiabetesManifestation empfohlen. Akutkomplikationen wie Hypoglykämien und diabetische Ketoazidose werden beim CFRD relativ selten beobachtet, letztere kommt aufgrund des meist nur relativen Insulinmangels so gut wie nie vor.

\section{Andere Diabetesformen}

Diese seltenen Erkrankungen umfassen infektiöse Formen (kongenitale Röteln) oder auch das StiffmanSyndrom (auch Stiff-Person-Syndrom genannt). Beim letzteren handelt es sich um eine Autoimmunerkrankung mit neurologischer Symptomatik, die spontan oder paraneoplastisch auftreten kann.

\section{Exokrine Pankreasinsuffizienz (EPI)}

\section{Prävalenz und Pathogenese}

In der Literatur werden EPI-Prävalenzen zwischen 10 und $56 \%$ bei Patienten mit Typ 1 Diabetes angegeben [59-63]. Eine schwere EPI, die sich definiert durch eine Elastase-1-Konzentration im Stuhl $<100 \mu \mathrm{g} / \mathrm{g}$, wurde dabei bei $10-30 \%$ der Patienten festgestellt. Eine EPI ist bei jedem dritten Patienten mit Typ 2 Diabetes beschrieben, wobei mehr als die Hälfte eine schwere Form aufweist [59, 60, 63-65]. Ursächlich findet sich bei DM1 eine verminderte Dichte an parasympathischen Axonen im exokrinen Pankreas [66], zudem kommt es im Rahmen der Entzündung auch zu einer Störung der Betazellregeneration, was aufgrund des gemeinsamen Ursprungs von exokrinen und endokrinen pankreatischen Vorläuferzellen ebenso zur exokrinen Insuffizienz beiträgt [67-69].

Die chronische Pankreatitis ist die häufigste Ursache der EPI bei Erwachsenen [70, 71]. EPI ist ein unabhängiger Risikofaktor für Mortalität bei chronischer Pankreatitis [72]. Etwa 85\% der Neugeborenen mit cystischer Fibrose (CF) sind pankreatisch insuffizient (PI) [73]. Neben Pankreasresektionen [74] können auch Magen- oder Dünndarmresektionen durch den Verlust der Sekretin- und Cholecystokinin Synthese bzw. rascher Magenentleerung mit EPI assoziiert sein, woran auch bei Patienten nach bariatrischer Chirurgie gedacht werden sollte [75]. Eine altersbedingte Pankreasatrophie (5\% ab >70 Jahren, $10 \%>80$ Jahren) kann ebenfalls mit EPI einhergehen [76].

\section{Klinische Manifestationen bei EPI}

Patienten mit milder EPI können asymptomatisch sein oder über leichtes abdominelles Unbehagen und Blähungen mit normalem Stuhlgang berichten. Bei schwerer EPI kann es infolge von Fett- und ProteinMaldigestion zu Gewichtsverlust kommen. Eine offenkundige Steatorrhoe tritt erst bei Verlust von ca. $90 \%$ der glandulären Funktion auf und geht mit übelriechenden, fettigen Stühlen mit reduzierter Konsistenz, die sich schwer wegspülen lassen, einher. Weitere Symptome sind Blähungen, abdominelle Krämpfe, Flatulenz. Obwohl klinisch symptomatische Vitaminmangelzustände mit metabolischer Knochenerkrankung oder gestörter Nachtsicht selten sind, sollte ein Mangel an fettlöslichen Vitaminen (ADEK) ausgeschlossen werden [71, 77-79]. Seltener liegt ein Vitamin B12 Mangel vor (reduzierter intestinaler $\mathrm{pH}$ ).

\section{Screening und Diagnostik der exokrinen Pankreasinsuffizienz}

Wegweisend ist hierzu die Anamnese. EPI sollte bei Patienten mit chronischer Diarrhoe/Steatorrhoe und chronischen Abdominalschmerzen, aber auch bei Patienten mit milderen Symptomen wie Blähungen und typischer Bildgebung für chronische Pankreatitis (z. B. Kalzifikationen und/oder Pankreasgangdilatationen und/oder Pseudozysten im Ultraschall, CT oder MRT) oder Pankreasatrophie suspiziert werden.

Bei entsprechender Symptomatik folgt zur weiteren Diagnostik meist ein indirekter (einfach, günstig) oder direkter Pankreas-Funktionstest (Stimulation des Pankreas durch hormonelle Sekretagoga mit nachfolgender Entnahme und Analyse von Duodenalflüssigkeit, z.B. Sekretin Test: aufwendig, invasiv, schlechte Patiententoleranz). Die Bestimmung der Elastase1-Konzentration im Stuhl mittels Immunassay (indirekter Test) gilt als Standardtest mit einer Sensitivität von etwa $65 \%$ bei milder und bis zu $100 \%$ bei schwerer Form [80]. Eine fäkale Elastase- $1<200 \mu \mathrm{g} / \mathrm{g}$ gilt als pathologisch, eine fäkale Elastase- $1<100 \mu \mathrm{g} / \mathrm{g}$ gilt als schwere EPI.

Aufgrund der relativ hohen Prävalenz der EPI ist bei gastrointestinaler Beschwerdesymptomatik ein Screening bei Patienten mit Diabetes sinnvoll [81-85].

Die differentialdiagnostisch in Erwägung zu ziehenden Erkrankungen ergeben sich aus der genannten Symptomatik der EPI und umfassen insbesondere die zahlreichen anderen Ursachen einer chronischen Diarrhoe. Speziell sind eine autonome Neuropathie des Magen-Darm-Traktes sowie gastrointestinale Nebenwirkungen oraler Antidiabetika (Metformin, Acarbose, Inkretinanaloga) zu erwägen. Auch Zuckeraustauschstoffe, wie die häufig verwendete Fruktose oder Sorbit, können bei Unverträglichkeit vergleichbare Symptome verursachen. Differentialdiagnostisch kommt auch eine häufig bei Diabetikern vorliegende 
bakterielle Fehlbesiedlung im Dünndarm in Betracht [86].

Speziell bei Typ I Diabetikern sollte an eine Zöliakie gedacht und diese mittels serologischer Diagnostik [endomysiale Antikörper (EMA), Antikörper gegen Gewebstransglutaminase (tTG) oder deamidiertes Gliadin Peptid (DGP)] ausgeschlossen werden. Ein normales Stuhl Calprotectin ist bei der Unterscheidung zwischen organischen/entzündlichen (z.B. M. Crohn, Colitis ulcerosa) und funktionellen gastrointestinalen Erkrankungen (z. B. Reizdarm, funktionelle Dyspepsie) hilfreich. Zusätzlich ist der Ausschluss eines Pankreaskarzinoms (mittels CT/MRT und/oder Endosonographie) wichtig, welches bei Diabetes im Vergleich zur nicht diabetischen Bevölkerung häufiger auftritt [87-89].

\section{Therapie}

Die Therapie der EPI besteht in einer dem Ausmaß der EPI sowie der Ernährung angepassten, ausreichenden Substitution von Pankreasenzymen. Bei schwerer Pankreasinsuffizienz sind pro Gramm Fett 2000 bis 4000 Einheiten Lipase nötig. Die Dosisfindung richtet sich nach den klinischen Beschwerden des Patienten mit dem Ziel der Beschwerdefreiheit. Eine Ernährungsberatung zum möglichst guten Einschätzen der Fettmenge in der Nahrung und zur Vorbeugung von Mangelzuständen sollte zusätzlich erfolgen. Die Evidenz für die Behandlung der Malabsorption durch mangelnde Pankreasenzymsekretion ist sehr gut [78, 79, 82, 89-94]. Fettlösliche Vitamine sollten zusammen mit Pankreasenzymen eingenommen werden. Von Nikotinkonsum sollte unbedingt abgeraten werden, da Rauchen einen unabhängigen Risikofaktor für EPI sogar bei Patienten ohne vorhergehende Pankreaserkrankung zu sein scheint [95].

Funding Open access funding provided by University of Innsbruck and Medical University of Innsbruck.

Interessenkonflikt S. Kaser hat von folgenden Unternehmen, die auch fördernde Mitglieder der ÖDG sind, Forschungsunterstützungen und/oder Honorare erhalten: AstraZeneca, Boehringer Ingelheim, Eli Lilly, Merck Sharp \& Dohme, Novartis, Novo Nordisk, Roche, sanofi-aventis, Servier, Takeda. Y. Winhofer-Stöckl hat von folgenden Unternehmen, die auch fördernde Mitglieder der ÖDG sind, Forschungsunterstützungen und/oder Honorare erhalten: Sanofi Aventis, AstraZeneca, Novartis, Eli Lilly. L. Kazemi-Shirazi hat Honorare von MSD, AbbVie, Ferring, MerckSerono/Dr Falk, Chiesi, Novartis, Roche, Abbott, Phadia Austria/Thermo Fisher Scientific und CSL-Behring sowie nicht-finanzielle Unterstützung von Mylan, Abbott, MSD, Gilead, MerckSerono/Dr Falk, Pfizer und Novartis erhalten. S. E. Hofer hat von folgendem Unternehmen, das auch förderndes Mitglied der ÖDG ist, Forschungsunterstützungen und/oder Honorare erhalten: Eli Lilly. H. Brath hat von folgenden Unternehmen, die auch fördernde Mitglieder der ÖDG sind, Forschungsunterstützungen und/oder Honorare erhalten: Abbott Diabetes Care, Amgen, AstraZeneca, Boehringer Ingelheim, Eli Lilly, Medtronic, MedMedia, MSD, Novartis, Novo Nordisk, Sanofi,
Servier. H. Sourij hat von folgenden Unternehmen, die auch fördernde Mitglieder der ÖDG sind, Forschungsunterstützungen und/oder Honorare erhalten: Amgen, AstraZeneca, Eli Lilly, Merck Sharp \& Dohme, Novo Nordisk, sanofi-aventis, Takeda. G. Vila und H. Abrahamian geben an, dass kein Interessenskonflikt besteht. M. Riedl hat von folgenden Unternehmen, die auch fördernde Mitglieder der ÖDG sind, Forschungsunterstützungen und/oder Honorare erhalten: AstraZeneca, Eli Lilly, Novo Nordisk, Novartis, Medronic, sanofi-aventis und Takeda sowie vom Bürgermeisterfond der Stadt Wien Proj.Nr.: 9073. R. Weitgasser hat von folgenden Unternehmen, die auch fördernde Mitglieder der ÖDG sind, Forschungsunterstützungen und/oder Honorare erhalten: Abbott, Allergan, AstraZeneca, Boehringer-Ingelheim, Dexcom, Eli Lilly, Merck Sharp \& Dohme, Novo Nordisk, Roche, sanofi-aventis, Servier, Takeda. M. Resl hat von folgenden Unternehmen, die auch fördernde Mitglieder der ÖDG sind, Forschungsunterstützungen und/oder Honorare erhalten: Boehringer Ingelheim, Eli Lilly, Novo Nordisk, sanofi-aventis, Amgen. M. Clodi hat von folgenden Unternehmen, die auch fördernde Mitglieder der ÖDG sind, Forschungsunterstützungen und/oder Honorare erhalten: AstraZeneca, Boehringer Ingelheim, Eli Lilly, Merck Sharp \& Dohme, Novartis, Novo Nordisk, sanofi-aventis, Servier, Takeda. A. Luger hat von folgenden Unternehmen, die auch fördernde Mitglieder der ÖDG sind, Forschungsunterstützungen und/oder Honorare erhalten: Novo Nordisk, Eli Lilly, Sanofi, Boehringer Ingelheim, MSD, Novartis.

Open Access Dieser Artikel wird unter der Creative Commons Namensnennung 4.0 International Lizenz (http:// creativecommons.org/licenses/by/4.0/deed.de) veröffentlicht, welche die Nutzung, Vervielfältigung, Bearbeitung, Verbreitung und Wiedergabe in jeglichem Medium und Format erlaubt, sofern Sie den/die ursprünglichen Autor(en) und die Quelle ordnungsgemäß nennen, einen Link zur Creative Commons Lizenz beifügen und angeben, ob Änderungen vorgenommen wurden.

Hinweis des Verlags Der Verlag bleibt in Hinblick auf geografische Zuordnungen und Gebietsbezeichnungen in veröffentlichten Karten und Institutsadressen neutral.

\section{Literatur}

1. Nieman LK, et al. The diagnosis of Cushing's syndrome: an Endocrine Society Clinical Practice Guideline. J Clin Endocrinol Metab. 2008;93(5):1526-40.

2. Nieman LK, et al. Treatment of Cushing's Syndrome: An Endocrine Society Clinical Practice Guideline. J Clin Endocrinol Metab. 2015;100(8):2807-31.

3. Katznelson L, et al. Acromegaly: an endocrine society clinical practice guideline. J Clin Endocrinol Metab. 2014;99(11):3933-51.

4. Frara S, et al. Current and Emerging Aspects of Diabetes Mellitus in Acromegaly. Trends Endocrinol Metab. 2016;27(7):470-83.

5. Abrahamian, H. et al. (2016) [Mental disorders and diabetes mellitus]. Wien Klin Wochenschr 128 [Suppl2]:S170-S178.

6. BreakeyS, etal. Glucocorticoid-induced hyperglycaemia in respiratory disease: a systematic review and meta-analysis. Diabetes Obes Metab. 2016;18(12):1274-8.

7. Katsuyama T, et al. Risk factors for the development of glucocorticoid-induced diabetes mellitus. Diabetes Res Clin Pract. 2015;108(2):273-9.

8. Penfornis, A. and Kury-Paulin, S. (2006) Immunosuppressive drug-induced diabetes. Diabetes Metab 32 (5 Pt 2), 539-46. 
9. van Raalte DH, et al. Novel insights into glucocorticoidmediated diabetogenic effects: towards expansion of therapeutic options? Eur JClin Invest. 2009;39(2):81-93.

10. Hecking M, et al. Glucose metabolism after renal transplantation. Diabetes Care. 2013;36(9):2763-71.

11. Kuo HT, et al. Risk factors for new-onset diabetes mellitus in adult liver transplant recipients, an analysis of the Organ Procurement and Transplant Network/United Network for Organ Sharing database. Transplantation. 2010;89(9):1134-40.

12. Schulte-Hermann K, et al. Impaired lipid profile and insulin resistance in a cohort of Austrian HIV patients. J Infect Chemother. 2016;22(4):248-53.

13. Cheema A, et al. Autoimmune Diabetes Associated With Pembrolizumab: A Review of Published Case Reports. World.JOncol.2018;9(1):1-4.

14. Tsiogka A, et al. Fulminant type 1 diabetes after adjuvant ipilimumab therapy in cutaneous melanoma. Melanoma Res. 2017;27(5):524-5.

15. Lee S, et al. Rapid-onset diabetic ketoacidosis secondary to nivolumab therapy. Endocrinol Diabetes Metab Case Rep. 2018. https://doi.org/10.1530/EDM-18-0021.

16. Elliott WJ, Meyer PM. Incident diabetes in clinical trials of antihypertensive drugs: a network meta-analysis. Lancet. 2007;369(9557):201-7.

17. Sattar N, et al. Statins and risk of incident diabetes: a collaborative meta-analysis of randomised statin trials. Lancet. 2010;375(9716):735-42.

18. Tattersall RB. Mild familial diabetes with dominant inheritance. QJ Med. 1974;43(170):339-57.

19. Tattersall RB, Fajans SS. A difference between the inheritance of classical juvenile-onset and maturity-onset type diabetes of young people. Diabetes. 1975;24(1):44-53.

20. Ellard S, et al. Best practice guidelines for the molecular genetic diagnosis of maturity-onset diabetes of the young. Diabetologia. 2008;51(4):546-53.

21. McDonaldTJ, EllardS. Maturity onset diabetes of theyoung: identification and diagnosis. Ann Clin Biochem 50. Pt, Bd. 5 . 2013. S. 403-15.

22. Stride A, Hattersley AT. Different genes, different diabetes: lessons from maturity-onset diabetes of the young. Ann Med.2002;34(3):207-16.

23. Martin D, et al. Long-term follow-up of oral glucose tolerance test-derived glucose tolerance and insulin secretion and insulin sensitivity indexes in subjects with glucokinase mutations (MODY2). Diabetes Care. 2008;31(7):1321-3.

24. Gardner DS, Tai ES. Clinical features and treatment of maturity onset diabetes of the young (MODY). Diabetes MetabSyndr Obes. 2012;5:101-8.

25. Feigerlova E, et al. Aetiological heterogeneity of asymptomatic hyperglycaemia in children and adolescents. Eur J Pediatr. 2006;165(7):446-52.

26. Velho G, et al. Identification of 14 new glucokinase mutations and description of the clinical profile of 42 MODY-2 families. Diabetologia. 1997;40(2):217-24.

27. Spyer G, et al. Influence of maternal and fetal glucokinase mutations in gestational diabetes. Am J Obstet Gynecol. 2001;185(1):240-1.

28. Hattersley AT, et al. Mutations in the glucokinase gene of the fetus result in reduced birth weight. Nat Genet. 1998;19(3):268-70.

29. Stride A, et al. Beta-cell dysfunction, insulin sensitivity, and glycosuria precede diabetes in hepatocyte nuclear factor-1alpha mutation carriers. Diabetes Care. 2005;28(7):1751-6
30. Pearson ER, et al. Genetic cause of hyperglycaemia and response to treatment in diabetes. Lancet. 2003;362(9392): 1275-81.

31. Pearson ER, et al. Molecular genetics and phenotypic characteristics of MODY caused by hepatocyte nuclear factor 4alpha mutations in a large European collection. Diabetologia.2005;48(5):878-85.

32. Pearson ER, et al. Macrosomia and hyperinsulinaemic hypoglycaemia in patients with heterozygous mutations in the HNF4Agene. Plos Med. 2007;4(4):e118.

33. EdghillEL, etal.Hepatocytenuclearfactor-1 betamutations cause neonatal diabetes and intrauterine growth retardation: support for a critical role of HNF-1beta in human pancreatic development. Diabet Med.2006;23(12):1301-6.

34. Fajans SS, et al. Molecular mechanisms and clinical pathophysiology of maturity-onset diabetes of theyoung. NEngl J Med.2001;345(13):971-80.

35. American Diabetes, A. (2014) Standards of medical care in diabetes-2014. Diabetes Care 37 Suppl 1, S14-80.

36. AmericanDiabetes, A. (2018) 2. Classification andDiagnosis of Diabetes: Standards of Medical Care in Diabetes-2018. Diabetes Care 41 (Suppl 1), S13-S27.

37. ReinauerC, etal.Lowprevalence of patients with mitochondrial disease in the German/Austrian DPV diabetes registry. Eur J Pediatr. 2016;175(5):613-22.

38. de Wit HM, et al. MIDD or MELAS : that's not the question MIDD evolving into MELAS : a severe phenotype of the m.3243A $>$ G mutation due to paternal co-inheritance of type 2 diabetes and a high heteroplasmy level. Neth J Med. 2012;70(10):460-2.

39. Tuomi T, et al. Atypical Forms of. Diabetes Endotext (de Groot LjEtAlEds).2000. PMID: 25905351.

40. El-Hattab, A.W. et al. (2015) MELAS syndrome: Clinical manifestations, pathogenesis, and treatment options. Mol Genet Metab 116 (1-2), 4-12.

41. Temple IK, Shield JP. 6q24 transient neonatal diabetes. Rev Endocr Metab Disord. 2010;11(3):199-204.

42. Wiedemann B, et al. Incidence of neonatal diabetes in Austria-calculation based on the Austrian Diabetes Register. Pediatr Diabetes. 2010;11(1):18-23.

43. Greeley SA, et al. Neonatal diabetes: an expanding list of genes allows for improved diagnosis and treatment. Curr DiabRep. 2011;11(6):519-32.

44. Babenko AP, et al. Activating mutations in the ABCC8 gene in neonatal diabetes mellitus. $\mathrm{N}$ Engl J Med. 2006;355(5):456-66.

45. Cui Y, Andersen DK. Pancreatogenic diabetes: special considerations for management. Pancreatology. 2011;11(3):279-94.

46. Ewald N, et al. Prevalence of diabetes mellitus secondary to pancreatic diseases (type 3c). Diabetes Metab Res Rev. 2012;28(4):338-42.

47. Andersen DK, et al. Pancreatogenic Diabetes, and Pancreatic Cancer. Diabetes. Diabetes. 2017;66(5):1103-10.

48. Slezak LA, Andersen DK. Pancreatic resection: effects on glucose metabolism. World J Surg. 2001;25(4):452-60.

49. Ewald N, Bretzel RG. Diabetes mellitus secondary to pancreatic diseases (Type 3c) - are we neglecting an important disease? Eur J Intern Med. 2013;24(3):203-6.

50. Ewald N, Hardt PD. Diagnosis and treatment of diabetes mellitus in chronic pancreatitis. World J Gastroenterol. 2013;19(42):7276-81.

51. Moran A, et al. Cystic fibrosis-related diabetes: current trends in prevalence, incidence, and mortality. Diabetes Care. 2009;32(9):1626-31.

52. KellyA, MoranA. Update on cystic fibrosis-related diabetes. JCyst Fibros. 2013;12(4):318-31. 
53. Boudreau V, et al. Variation of glucose tolerance in adult patients with cystic fibrosis: What is the potential contribution of insulin sensitivity? J Cyst Fibros. 2016;15(6):839-45.

54. Milla CE, et al. Diabetes is associated with dramatically decreased survival in female but not male subjects with cystic fibrosis. Diabetes Care. 2005;28(9):2141-4.

55. Lewis C, et al. Diabetes-related mortality in adults with cystic fibrosis. Role of genotype and sex. Am J Respir Crit Care Med. 2015;191(2):194-200.

56. Moran A, et al. Clinical care guidelines for cystic fibrosisrelated diabetes: a position statement of the American Diabetes Association and a clinical practice guideline of the Cystic Fibrosis Foundation, endorsed by the Pediatric Endocrine Society. Diabetes Care. 2010;33(12):2697-708.

57. Sunni M, et al. Exogenous insulin requirements do not differ between youth and adults with cystic fibrosis related diabetes. Pediatr Diabetes. 2013;14(4):295-8.

58. Balzer BW, et al. Low glycaemic index dietary interventions in youth with cystic fibrosis: a systematic review and discussion of the clinical implications. Nutrients. 2012;4(4):286-96.

59. Hardt PD, et al. Chronic pancreatitis and diabetes mellitus. A retrospective analysis of 156 ERCP investigations in patients with insulin-dependent and non-insulin-dependent diabetes mellitus. Pancreatology. 2002;2(1):30-3.

60. Hardt PD, et al. High prevalence of steatorrhea in 101 diabetic patients likely to suffer from exocrine pancreatic insufficiency according to low fecal elastase 1 concentrations: a prospective multicenter study. Dig Dis Sci. 2003;48(9):1688-92.

61. Icks A, et al. Low fecal elastase- 1 in type I diabetes mellitus. ZGastroenterol.2001;39(10):823-30.

62. Lankisch PG, et al. Exocrine pancreatic function in insulindependent diabetes mellitus. Digestion. 1982;25(3):211-6.

63. Piciucchi $M$, et al. Exocrine pancreatic insufficiency in diabetic patients: prevalence, mechanisms, and treatment. Int JEndocrinol.2015;2015:595649.

64. Bretzke G. Diabetes mellitus and exocrine pancreas function.ZGesamteInn Med. 1984;39(16):388-90.

65. Rathmann W, et al. Low faecal elastase 1 concentrations in type 2 diabetes mellitus. Scand J Gastroenterol. 2001;36(10):1056-61.

66. Lundberg $\mathrm{M}$, et al. The density of parasympathetic axons is reducedin the exocrinepancreas ofindividuals recentlydiagnosed with type 1 diabetes. PLoSONE. 2017;12(6):e179911.

67. Apte M, et al. The fibrosis of chronic pancreatitis: new insights into the role of pancreatic stellate cells. Antioxid RedoxSignal.2011;15(10):2711-22.

68. Hayden MR, et al. Attenuation of endocrine-exocrine pancreatic communication in type 2 diabetes: pancreatic extracellular matrix ultrastructural abnormalities. J Cardiometab Syndr. 2008;3(4):234-43.

69. Wu D, et al. Endocrine pancreatic function changes after acute pancreatitis. Pancreas. 2011;40(7):1006-11.

70. Bellin MD, et al. Patient and Disease Characteristics Associated With the Presence of Diabetes Mellitus in Adults With Chronic Pancreatitis in the United States. Am J Gastroenterol.2017;112(9):1457-65.

71. LohrJM, etal. UnitedEuropean Gastroenterology evidencebased guidelines for the diagnosis and therapy of chronic pancreatitis (HaPanEU). United Eur Gastroenterol J. 2017;5(2):153-99.

72. Iglesia-Garcia D de la, et al. Increased Risk of Mortality Associated With Pancreatic Exocrine Insufficiency in Patients With Chronic Pancreatitis. J Clin Gastroenterol. 2018; 52(8):e63-e72. https://doi.org/10.1097/MCG. 0000000000000917 .
73. Van de Vijver E, et al. Treatment of infants and toddlers with cystic fibrosis-related pancreatic insufficiency and fat malabsorption with pancrelipase MT. J Pediatr Gastroenterol Nutr. 2011;53(1):61-4.

74. Roeyen G, et al. The impact of pancreaticoduodenectomy on endocrine and exocrine pancreatic function: A prospective cohort study based on pre- and postoperative function tests. Pancreatology. 2017;17(6):974-82.

75. Vujasinovic M, et al. Pancreatic Exocrine Insufficiency after BariatricSurgery. Nutrients. 2017.https://doi.org/10.3390/ nu9111241.

76. Lohr JM, et al. The ageing pancreas: a systematic review of the evidence and analysis of the consequences. J Intern Med.2018;283(5):446-60.

77. Moran CE, et al. Bone mineral density in patients with pancreatic insufficiency and steatorrhea. Am J Gastroenterol. 1997;92(5):867-71.

78. Sikkens EC, et al. The prevalence of fat-soluble vitamin deficiencies and a decreased bone mass in patients with chronic pancreatitis. Pancreatology. 2013;13(3):238-42.

79. Toouli J, et al. Management of pancreatic exocrine insufficiency: Australasian Pancreatic Club recommendations. Med JAust. 2010;193(8):461-7.

80. Loser C, et al. 13C-starch breath test-comparative clinical evaluation of an indirect pancreatic function test. Z Gastroenterol. 1997;35(3):187-94.

81. Cavalot F, et al. Does pancreatic elastase-1 in stools predict steatorrhea in type 1 diabetes? Diabetes Care. 2006;29(3):719-21.

82. Ebert R, Creutzfeldt W. Reversal of impaired GIP and insulin secretion in patients with pancreatogenic steatorrhea following enzyme substitution. Diabetologia. 1980;19(3):198-204.

83. Ewald, N. et al. (2009) Determinants of Exocrine Pancreatic Function as Measured by Fecal Elastase-1 Concentrations (FEC) in Patients with Diabetes mellitus. Eur J Med Res 14 (3), 118-22.

84. Hardt PD, et al. High prevalence of exocrine pancreatic insufficiency in diabetes mellitus. A multicenter study screening fecal elastase 1 concentrations in 1,021 diabetic patients. Pancreatology. 2003;3(5):395-402.

85. Nunes AC, et al. Screening for pancreatic exocrine insufficiency in patients with diabetes mellitus. Am J Gastroenterol.2003;98(12):2672-5.

86. Ni Chonchubhair HM, et al. The prevalence of small intestinal bacterial overgrowth in non-surgical patients with chronic pancreatitis and pancreatic exocrine insufficiency (PEI). Pancreatology. 2018;18(4):379-385. https://doi.org/ 10.1016/j.pan.2018.02.010.

87. Keller, J. et al. (1997) Duodenal and ileal nutrient deliveries regulate human intestinal motor and pancreatic responses to a meal. Am J Physiol 272 (3 Pt 1), G632-7.

88. Layer P, et al. Altered postprandial motility in chronic pancreatitis: role of malabsorption. Baillieres Clin Gastroenterol. 1997;112(5):1624-34.

89. Leeds JS, et al. The role of fecal elastase- 1 in detecting exocrine pancreatic disease. Nat Rev Gastroenterol Hepatol. 2011;8(7):405-15.

90. Dominguez-Munoz JE. Pancreatic enzyme replacement therapy for pancreatic exocrine insufficiency: when is it indicated, what is the goal and how to do it? Adv Med Sci. 2011;56(1):1-5.

91. Dominguez-Munoz JE. Chronic pancreatitis and persistent steatorrhea: what is the correct dose of enzymes? Clin Gastroenterol Hepatol.2011;9(7):541-6.

92. Gubergrits, N. et al. (2011) A 6-month, open-label clinical trial of pancrelipase delayed-release capsules (Creon) in pa- 


\section{leitlinien für die praxis}

tients with exocrine pancreatic insufficiency due to chronic pancreatitis or pancreatic surgery. Aliment PharmacolTher 33 (10), 1152-61.

93. Sikkens EC, et al. Pancreatic enzyme replacement therapy in chronic pancreatitis. Best Pract Res Clin Gastroenterol. 2010;24(3):337-47.

94. de la Iglesia-Garcia D, et al. Efficacy of pancreatic enzyme replacement therapy in chronic pancreatitis: systematic review and meta-analysis. Gut. 2017;66(8):1354-5.

95. Raphael KL, et al. Pancreatic Insufficiency Secondary to Tobacco Exposure: A Controlled Cross-Sectional Evaluation. Pancreas. 2017;46(2):237-43.

96. Sarafidis PA, Bakris GL. Antihypertensive treatment with beta-blockers and the spectrum of glycaemic control. QJM. 2006;99(7):431-6.

97. Luna B, Feinglos MN. Drug-induced hyperglycemia. JAMA. 2001;286(16):1945-8.
98. Thomas, Z. et al. (2010) Drug-induced endocrine disorders in the intensive care unit. Crit Care Med 38 (6 Suppl), S219-30.

99. Fuhrmann A, et al. Molecular mechanisms underlying the effects of cyclosporin A and sirolimus on glucose and lipid metabolism in liver, skeletal muscle and adipose tissuein an in vivo rat model. Biochem Pharmacol. 2014;88(2):216-28.

100. Verges B. Effects of anti-somatostatin agents on glucose metabolism. Diabetes Metab. 2017;43(5):411-5.

101. Watt KD, Charlton MR. Metabolic syndrome and liver transplantation: a review and guide to management. J Hepatol. 2010;53(1):199-206.

102. De Hert M, et al. Metabolic and cardiovascular adverse effects associated with antipsychotic drugs. Nat Rev Endocrinol.2011;8(2):114-26. 